Article

\title{
Numerical and Experimental Investigations on Vocal Fold Approximation in Healthy and Simulated Unilateral Vocal Fold Paralysis
}

\author{
Zheng Li ${ }^{1}{ }^{1}$, Azure Wilson ${ }^{2}$, Lea Sayce ${ }^{2}$, Amit Avhad ${ }^{1}$, Bernard Rousseau ${ }^{2, *}$ and Haoxiang Luo ${ }^{1, *}$ \\ 1 Department of Mechanical Engineering, Vanderbilt University, 2301 Vanderbilt Place, PMB 401592, \\ Nashville, TN 37240, USA; zheng.li@vanderbilt.edu (Z.L.); amit.g.avhad@vanderbilt.edu (A.A.) \\ 2 Department of Communication Science and Disorders, University of Pittsburgh, 4200 Fifth Avenue, \\ Pittsburgh, PA 15260, USA; azure.wilson@pitt.edu (A.W.); lea.sayce@pitt.edu (L.S.) \\ * Correspondence: rousseau.csd@pitt.edu (B.R.); Haoxiang.luo@vanderbilt.edu (H.L.)
}

Citation: Li, Z.; Wilson, A.; Sayce, L.; Avhad, A.; Rousseau, B.; Luo, H. Numerical and Experimental Investigations on Vocal Fold Approximation in Healthy and Simulated Unilateral Vocal Fold Paralysis. Appl. Sci. 2021, 11, 1817. https://doi.org/10.3390/app11041817

Academic Editor: Michael Döllinger Received: 31 December 2020

Accepted: 10 February 2021

Published: 18 February 2021

Publisher's Note: MDPI stays neutral with regard to jurisdictional claims in published maps and institutional affiliations.

Copyright: (c) 2021 by the authors. Licensee MDPI, Basel, Switzerland. This article is an open access article distributed under the terms and conditions of the Creative Commons Attribution (CC BY) license (https:// creativecommons.org/licenses/by/ $4.0 /)$.

\begin{abstract}
We have developed a novel surgical/computational model for the investigation of unilateral vocal fold paralysis (UVFP) which will be used to inform future in silico approaches to improve surgical outcomes in type I thyroplasty. Healthy phonation (HP) was achieved using cricothyroid suture approximation on both sides of the larynx to generate symmetrical vocal fold closure. Following high-speed videoendoscopy (HSV) capture, sutures on the right side of the larynx were removed, partially releasing tension unilaterally and generating asymmetric vocal fold closure characteristic of UVFP (sUVFP condition). HSV revealed symmetric vibration in HP, while in sUVFP the sutured side demonstrated a higher frequency (10-11\%). For the computational model, ex vivo magnetic resonance imaging (MRI) scans were captured at three configurations: non-approximated (NA), HP, and sUVFP. A finite-element method (FEM) model was built, in which cartilage displacements from the MRI images were used to prescribe the adduction, and the vocal fold deformation was simulated before the eigenmode calculation. The results showed that the frequency comparison between the two sides was consistent with observations from HSV. This alignment between the surgical and computational models supports the future application of these methods for the investigation of treatment for UVFP.
\end{abstract}

Keywords: voice production; unilateral vocal fold paralysis; cricothyroid suture approximation; finite element modeling; vocal fold eigenfrequency

\section{Introduction}

The vocal folds are a pair of highly specialized tissues housed in the larynx, which when adducted into glottal controlled airflow, vibrate to produce voice. In humans, the vocal folds consist of five distinct layers; luminal stratified squamous epithelia, three lamina propria layers, and a basal ligament (vocalis muscle), which are broadly segmented into a cover and body as previously described [1]. Disordered vocal folds lead to many impairments, including poor voice quality, swallowing dysfunction, breathlessness, and aspiration [2]. Unilateral vocal fold paralysis (UVFP) is a voice disorder caused by injury of the recurrent laryngeal nerve or vagus nerve innervating the larynx [3], resulting in impaired adduction, one-sided vocal fold immobility, and a widened glottal gap. UVFP has an iatrogenic origin in around half of all cases [4], most frequently as a result of thyroidectomy or anterior cervical disk spinal fusion, but may also present idiopathically [5]. The symptomatic burden of UVFP on patients is significant with $53-100 \%$ of identified cases experiencing dysphonia [6-8], 60-75\% dyspnea [9,10], and approximately 60\% dysphagia [6], resulting in an increased risk of aspiration [11].

Surgical intervention is necessary for more than half of patients diagnosed with UVFP, with a primary goal of medializing the affected vocal fold to correct the glottal insufficiency [10]. There are two commonly used techniques for medialization laryngoplasty. 
Injection laryngoplasty, a technique for which the vocal folds are medialized using an autologous or biologically inert substance that is injected directly into the thyroarytenoid muscle, is used to improve short-term voice outcomes (typically weeks to months depending on material and resorption rate) in patients who may experience spontaneous recovery or for whom more invasive surgery or general anesthesia is contraindicated [12-18]. Type I thyroplasty, a technique with which medialization is achieved by surgical insertion of a bio-tolerable implant (e.g., Silastic) or a stacked Gore-Tex ribbon through a window in the thyroid cartilage, provides longer duration results, but as with injection laryngoplasty, it is not without limitations, including: under/over medialization, size and shape variability when hand-carving implants, Gore-Tex compression over time, and inflammatory reactions. Inadequate medialization is reported relatively frequently, with $4.5-16 \%$ of type I thyroplasty procedures requiring surgical revision [19-23].

Previous efforts to surgically investigate type I thyroplasty are largely limited to studies in cadaver larynges assessing changes in biomechanics following implantation, or to histological analyses of larynges post-implantation; however, several in vivo and clinical studies evaluating phonatory measures have been described. In a canine model, Green et al. [24] simulated UVFP by cuffing the recurrent and superior laryngeal nerves and identified that arytenoid adduction + type I thyroplasty using the Hiroto method [25] increased vocal efficiency significantly more than augmentation alone, although the authors acknowledge that the longer posterior glottal chink in dogs vs. humans likely contributes to less optimal outcomes from implantation. Another study in a canine model identified significant impacts of both shape and medialization depth on fundamental frequency and aerodynamic power required for phonation and was also able to demonstrate reduced efficacy of type I thyroplasty in vagal paralysis vs. recurrent laryngeal nerve paralysis [26]. In a clinical population, type I thyroplasty in combination with cricothyroid suture approximation (also known as type IV thyroplasty) was demonstrated to improve video- stroboscopic, acoustic perceptual, and subjective dysphonia ratings in patients with unilateral superior laryngeal nerve weakness [27]. In a separate patient population with unilateral cricothyroid muscle paralysis, Shaw et al. reported that cricothyroid suture approximation alone was sufficient to improve multiple measures including glottic closure, frequency (fundamental, falsetto, basal), maximum phonatory time, and patient-reported perception [28] Subsequent investigation of cricothyroid suture approximation by our group in a rabbit model allowed for the development of a non-stimulatory method for medialization which achieved fundamental frequencies, vocal intensities, and aerodynamic measures consistent with modal phonation elicited through electrical stimulation [29]. The cricothyroid suture approximation model described by Novaleski et al. has the specific advantage of retaining vocal fold approximation ex vivo for imaging studies, something that cannot be achieved by electrical stimulation, such that high-speed videoendoscopy (HSV) and imaging from the same subject can be used to inform computational models of vocal fold vibration [30].

Besides experimental efforts, many numerical investigations using computational mechanics models have been conducted to study vocal fold vibration. Complementary to the physical models in experimental studies, computational models have the advantage of providing insight into the phonation process and predicting the vibration through mathematical modeling and computer simulation of the physical system. Such computational models usually include governing equations for the vocal fold biomechanics, the glottal aerodynamics, or a combination of both for fluid-structure interaction (FSI) simulations. Depending on the realism of vocal fold representation, there were lumpedmass models, two-dimensional, and three-dimensional finite-element models of the vocal fold tissues [30-36]. With the fast growth of computational power and medical imaging technologies, e.g., computed tomography (CT) and magnetic resonance imaging (MRI), patient-specific geometries have been incorporated into the FEM model of the vocal fold to represent the anatomy of individual patients or subjects [37-39]. Such models have potential applications in the surgical treatment of voice disorders like UVFP. More details about the development of computational models can be found in recent review papers $[38,40]$. 
While simulating vocal fold vibration, many FEM models have ignored the vocal fold adduction process and assumed equivalent stiffness properties for the vocal fold tissue to produce a suitable fundamental frequency. This ad hoc assumption may include asymmetric vibration, where two sides were assigned with different stiffness properties [41]. To study the effect of the approximation on vibration, the large tissue deformation due to adduction should be part of the FEM model to account for the effect of the structural stiffening (as opposed to material stiffening) on the dynamic behavior of the vocal fold. In the past, quite a few FEM models have been developed in combination with experimental testing to investigate vocal fold adduction, including both in vivo and ex vivo conditions [42-48], while the main interests of these studies were to determine the material properties of the vocal fold tissue. Some studies have looked at the effect of adduction or lengthening on the vocal fold structure $[42,45,46]$. However, those models only have simple vocal fold geometries and have not included details of cartilage movement. To accurately simulate vocal fold approximation, it is necessary to obtain quantitative information of cartilage displacement in the process. There have been efforts to segment and reconstruct laryngeal cartilages based on 3D image data [49,50]; however, this approach has not been used in FEM modeling of the vocal fold.

In this study, we constructed a subject-specific rabbit vocal fold model based on the excised MRI scans at different surgical vocal fold approximation conditions. From these scans including that from the non-approximated (NA) condition, we obtained the details of cartilage displacement and incorporated them into the FEM model as the boundary condition to simulate the vocal fold deformation and consequent structural stiffening. Compared with previous studies, our FEM model incorporates the subject-specific anatomy and the displacement of the cartilage from the MRI data. Furthermore, previous works predominantly focused on normal healthy vocal fold adduction, with the two sides producing symmetrical vibration. In the current study, we considered a simulated UVFP condition (sUVFP), in which the two vocal folds were asymmetrically approximated, and built the corresponding FEM model that has different structural stiffening due to deformation between the two sides. This model allows us to study the effect of the asymmetric adduction on the vocal fold vibration without having to make ad hoc assumptions of different material stiffness properties for the two sides.

In this work, we combined computational modeling with the experimental study of multiple rabbit larynges, and by comparing healthy phonation with simulated UVFP (sUVFP) we investigated the effect of the sUVFP on vocal fold vibration. The experimental data will validate the computational model, and the computational model will provide insight into the experimental observation in terms of vibration asymmetry. This combined experimental/computational approach and its alignment are demonstrated in this sUVFP study. Similar approaches will be utilized in the future to study the treatment approach for the UVFP.

\section{Materials and Methods}

\subsection{Surgical Methodology}

\subsubsection{Animals}

This study was approved by the University of Pittsburgh Institutional Animal Care and Use Committee (IACUC \#18114069). All procedures were conducted within the University of Pittsburgh Department of Laboratory Animal Research, an AAALAC-accredited facility, in compliance with the Guide for the Care and Use of Laboratory Animals and the Animal Welfare Regulations [51,52]. Six New Zealand white breeder rabbits were included in this study, three for in vivo phonation studies with HSV analyses, and three for ex vivo MRI studies. The anesthesia regimen has been described elsewhere [53]. Briefly, rabbits were sedated with $20 \mathrm{mg} / \mathrm{kg}$ ketamine and $0.125 \mathrm{mg} / \mathrm{kg}$ of dexmedetomidine by intramuscular injection. To maintain anesthesia (as required), continuous rate infusion of $350 \mu \mathrm{g} / \mathrm{kg} / \mathrm{min}$ ketamine and $1.65 \mu \mathrm{g} / \mathrm{kg} / \mathrm{min}$ dexmedetomidine was provided intra- 
venously, via $27 \mathrm{G}$ catheter to the marginal vein. Heart rate, respiratory rate, temperature, and oxygen partial pressure were recorded for the duration of the procedure.

\subsubsection{In Vivo Phonation}

Non-stimulated phonation was performed as previously described [29] with minor modifications. In brief, the neck and chest of anesthetized rabbits were shaved and the rabbit was arranged in a supine position. A skin incision was made from sternum to submentum, and the fascia and muscle were dissected at the midline to expose the larynx and trachea. The trachea was bisected and an uncuffed endotracheal tube was inserted into the arboreal portion to provide direct oxygen to the lungs. A cuffed endotracheal tube was positioned in the upper portion of the trachea and provided humidified airflow at $37^{\circ} \mathrm{C}$ to the subglottis. A pediatric laryngoscope was inserted via the mouth to suspend the larynx and visualize the vocal folds, and a combination of humidified airflow and cricothyroid suture approximation (see Section 2.1.3 for laryngeal conditions) was used to achieve phonation (see Figure 1). Phonation was not achieved in the NA condition. HSV was captured at 8000 frames per second in each laryngeal condition using a KayPENTAX FastCam MC2.1 system coupled to a $0^{\circ}$ rigid endoscope.

(a)

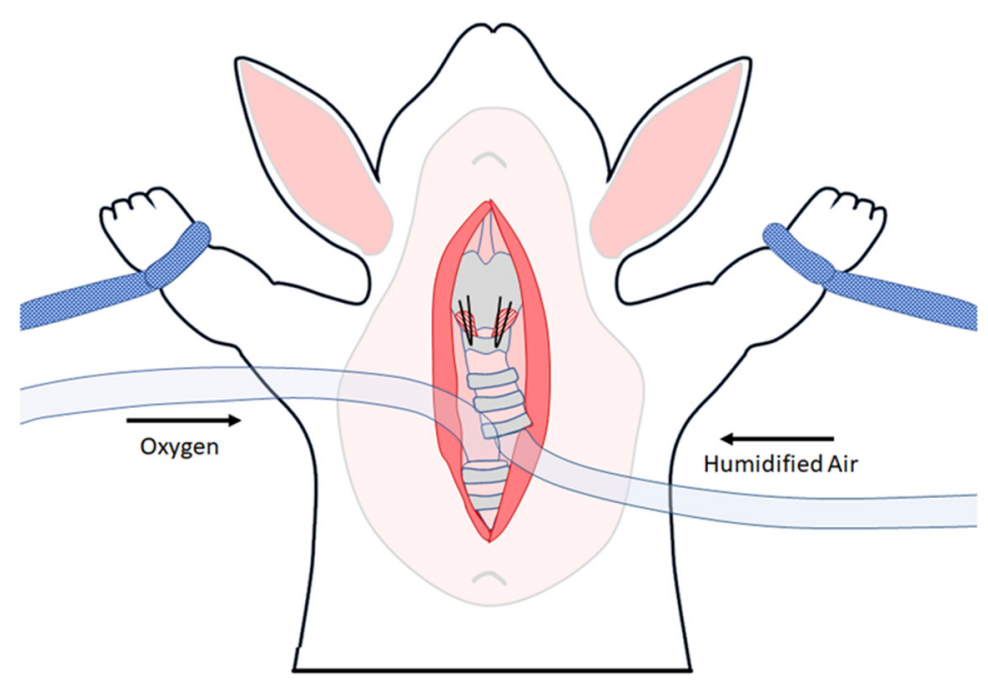

(b)
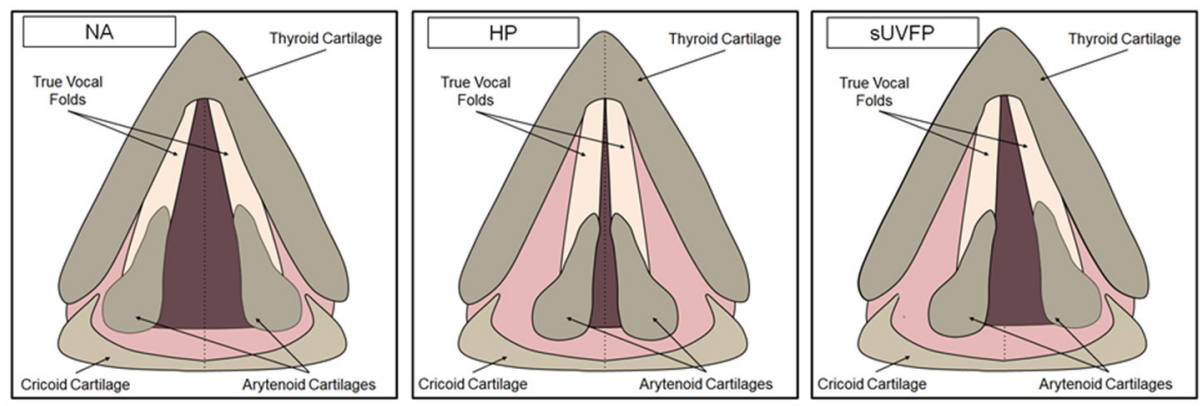

Figure 1. Non-stimulated phonation methodology. (a) Pictorial representation of non-stimulated vocal fold phonation using bilateral sutures (HP condition) to achieve vocal fold approximation in a rabbit model. The trachea was bisected and humidified airflow is supplied to the subglottis, in combination with bilateral cricothyroid suture approximation to move the vocal folds toward the midline, resulting in phonation. (b) Pictorial representation of endoscopic vocal fold views captured by HSV in three experimental conditions: non-approximated (NA), healthy phonation (HP), and simulated unilateral vocal fold paralysis (sUVFP). 


\subsubsection{Cricothyroid Suture Approximation Laryngeal Conditions}

Cricothyroid suture approximation was performed as previously described [29] with minor modifications to achieve NA, HP, and sUVFP laryngeal conditions. Conditions were performed in a stepwise fashion to capture all conditions in each larynx (Figure 2). NA: HSV of the vocal folds was captured in a neutral resting position. HP: Bilateral Vicryl 5.0 sutures (Ethicon) were used to suture the cricoid cartilage to the thyroid cartilage, resulting in lengthening of both vocal folds to approximate them medially. sUVFP: Sutures were removed from the right side of the larynx, resulting in tension being released from the right vocal fold and a "floppy" appearance characteristic of UVFP.
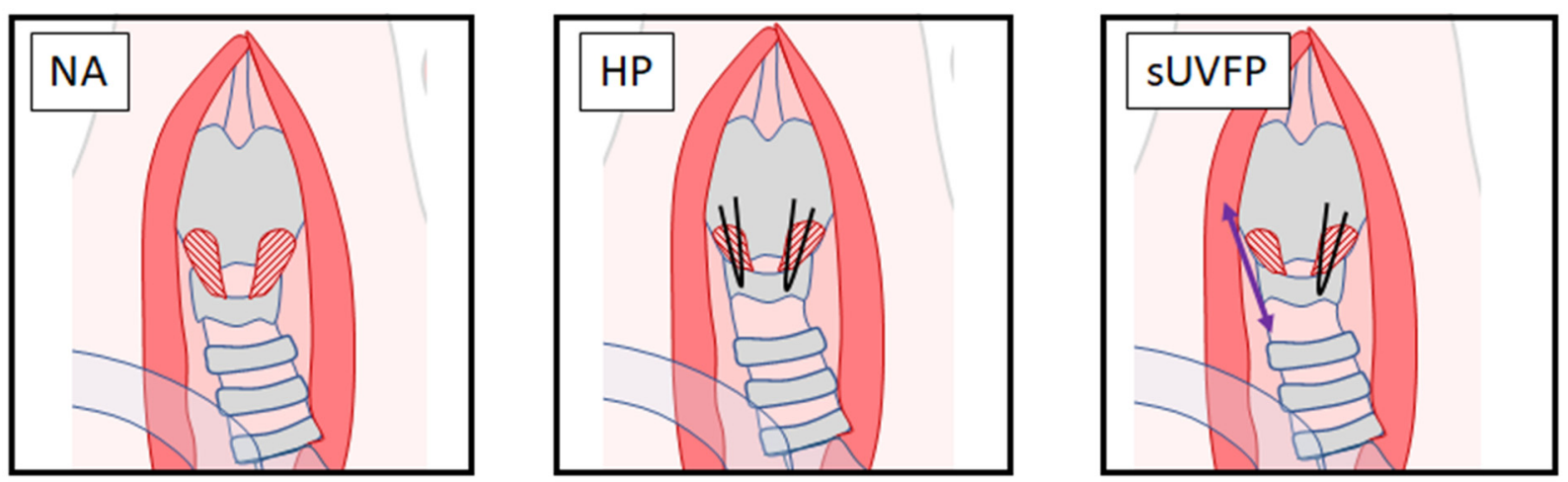

Figure 2. Cricothyroid suture approximation conditions. HSV was captured in three conditions in vivo. The NA condition was captured before suturing to observe the natural resting vocal fold position. The HP condition was captured following bilateral sutures between the cricoid and thyroid cartilages, shortening the distance between these structures and lengthening the vocal folds to achieve approximation. The sUVFP condition was captured by releasing the right-sided sutures, allowing lengthening of the distance between the cricoid and thyroid unilaterally, leading to the right vocal fold having less tension applied and appearing "floppy".

\subsubsection{Ex Vivo Cricothyroid Suture Approximation and MRI}

Larynges were harvested for MRI in animals that had received no prior interventions. Excised larynges were scanned in the following experimental conditions: NA, HP, sUVFP (see Figure 2). Excised larynges were placed in a $10 \mathrm{~mL}$ syringe with perfluorocarbon oil and images were captured with a Bruker AV3HD 11.7 tesla/89 mm vertical-bore micro-imaging system equipped with a 40-mm micro2.5 set capable of $1500 \mathrm{mT} / \mathrm{m}$ and ParaVision 6.0.1 software (Bruker Biospin). Three-dimensional T2-weighted images were collected at an isotropic resolution of $60 \mu$ using a fast spin-echo sequence with the following parameters: TR/TE $1000 / 28$ ms, $256 \times 384 \times 286$ matrix, $16 \times 16$ mm field-of-view, and a RARE factor of 8 .

\subsection{Numerical Methodology}

To numerically investigate the effect of vocal fold approximation on vibration in different conditions, we first reconstructed subject-specific larynges based on ex vivo experimentation. Due to the duration of the MRI scans to obtain appropriate resolution for computational modeling (approximately $4 \mathrm{~h}$ per scan) we are not able to gather this data in vivo due to concerns of protracted anesthesia, movement during scans, and general welfare concerns. It was not practicable to use larynges previously used for in vivo experimentation due to tissue inflammation and damage caused by phonation, such that tissue structure was likely to be altered by the time MRI imaging was performed. Therefore, three additional rabbit larynges were utilized for the model reconstructions.

\subsubsection{Vocal Fold Reconstruction from MRI Scan}

Computational laryngeal reconstruction was performed using MRI images from three samples that were scanned ex vivo. Using sample 1 as an example (see Figure 3), we first scanned the vocal fold in the NA condition. Following this, the MRI scans were 
imported to ITK-SNAP to manually segment the images. We considered the following structural components for model reconstruction: thyroid cartilage, arytenoid cartilages, cricoid cartilage, and soft tissue (vocal fold). Two distinct layers were segmented within the vocal folds due to known differences in tissue properties [54]: the pliable vocal fold cover, composed of the epithelium and superficial lamina propria, and the much stiffer vocal fold body, composed of the deep lamina propria and thyroarytenoid muscles. The layers were segmented in MRI images as depicted in Figure 3.

(a)

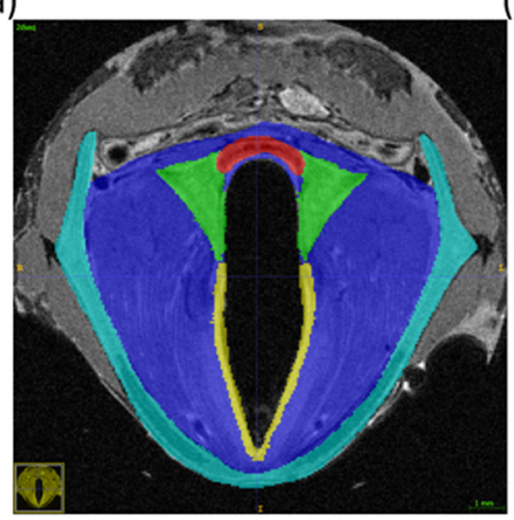

(b)

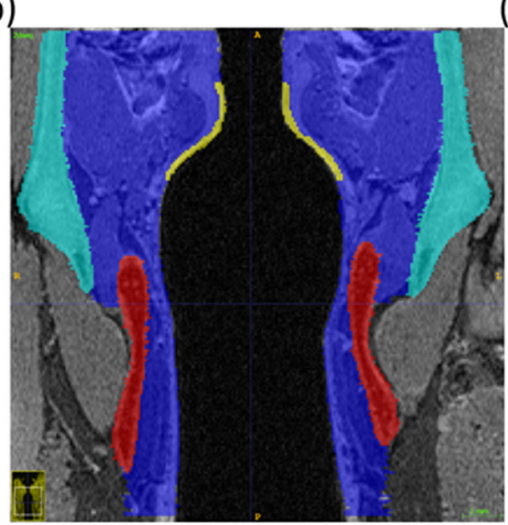

(c)

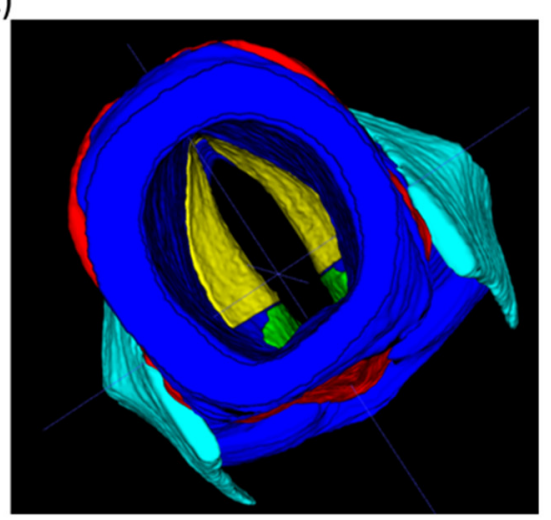

Figure 3. Vocal fold segmentation for sample 1. Green = arytenoid cartilages; red = cricoid cartilage; cyan = thyroid cartilage; blue = vocal fold body; yellow = vocal fold cover. (a) axial section; (b) coronal section; (c) 3D view from trachea.

Following segmentation, we output surface meshes for each structural component from ITK-SNAP and processed them with Mesh Mixer (Autodesk, San Francisco, CA, USA) to avoid uneven surfaces from the manual segmentation. However, this procedure leads to a separate surface mesh for each segmented component, and the two components adjacent to each other, e.g., the thyroid cartilage and the vocal fold body do not share the same surface on their meshes, and there exists a thin gap in between. To handle this problem, we output a surface mesh for the larynx as an entire segment from ITK-SNAP, and this mesh was also smoothed with MESH Mixer. Next, all the smoothed surface meshes are imported into COMSOL Multiphysics (COMSOL Inc., Burlington, MA, USA) to generate solid bodies and create unstructured volume meshes sequentially. A tetrahedron mesh with 10 nodes on each element was applied. Different structural components need to be identified on the entire larynx mesh as this mesh will be used as the FEM model in the following simulations. To do so, we compared the nodes on the volume mesh of the entire larynx with the nodes on the volume mesh of individual structural components and set a minimum distance criterion to determine whether the nodes of the entire larynx's mesh belong to a specific structural component. Using this approach, all the nodes on the entire larynx's mesh were marked as individual structural components as shown in Figure 4a and can be assigned with the material properties related to that component. Since the vocal fold cover is thin but important for the vocal fold vibration, this region is refined with denser mesh as shown in Figure $4 \mathrm{~b}$. In the mesh-independence study, four different unstructured meshes with 76,181, 88,334, 125,351, and 135,748 elements respectively were used to compute the eigenfrequency of approximated vocal folds in the HP condition. The eigenfrequency of the left vocal fold is $578 \mathrm{~Hz}, 588 \mathrm{~Hz}, 597 \mathrm{~Hz}$, and $598 \mathrm{~Hz}$, respectively, using the aforementioned four meshes. The third mesh resolution was selected in all the cases, as further refinement did not significantly improve the result. In this mesh set, the thyroid cartilage, arytenoid cartilage, cricoid cartilage, vocal fold cover, and vocal fold body for Sample 1 have 16,510, 3232, 11,489, 13,021, and 81,099 elements respectively. 
(a)

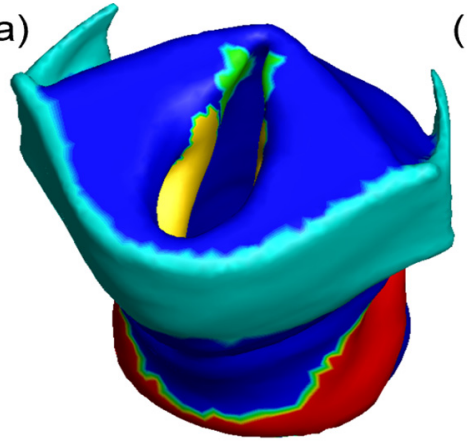

(b)

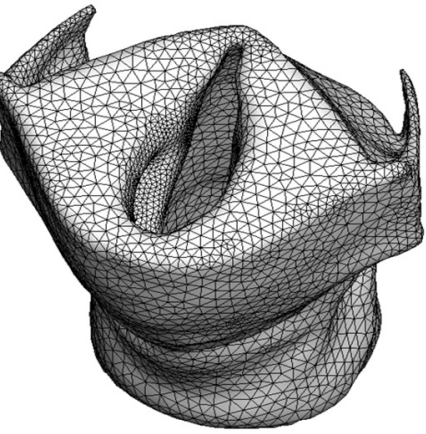

Figure 4. Reconstructed vocal fold from Sample 1. (a) Reconstructed vocal fold, whose details are shown in Figure 3. (b) Unstructured mesh for reconstructed vocal fold, where the vocal fold cover is refined.

\subsubsection{Vocal Fold Approximation Modeling}

Once the NA larynx was reconstructed in Section 2.2.1, we were able to model vocal fold approximation using sutures in the HP and sUVFP conditions. Following the MRI scan for the NA condition, the HP and sUVFP cricothyroid suture approximation conditions were implemented in a stepwise fashion with consecutive MRI imaging.

Images of the HP condition were manually segmented and reconstructed with ITKSNAP. Figure 5a,b show the reconstructed thyroid cartilage, arytenoid cartilages, and cricoid cartilage in NA and HP conditions, respectively. MRI imaging of the sUVFP condition was also segmented and reconstructed manually with ITK-SNAP. Laryngeal changes between HP and sUVFP are represented from Figure $5 b$ to Figure $5 c$. A comparison of the reconstructed laryngeal conditions revealed that the cricoid cartilage retains the same spatial orientation and shape between the conditions, so it was used as a reference to calculate the thyroid cartilage displacement from NA to HP, and from NA to sUVFP. These displacements were prescribed to the mesh nodes on the thyroid cartilage in the reconstructed whole larynx model shown in Figure 5. The cricoid cartilage remained fixed in the simulations. The Saint Venant-Kirchhoff model was adopted to represent the tissue properties for the vocal fold body and cover. With this model, we assume the vocal fold tissue to be isotropic with a linear relationship between the stress and the strain. However, the finite strain takes into account the nonlinear effects due to large displacements and large rotation $[55,56]$. Details for this hyper-elastic model can be found in our previous works [31]. With this procedure, we were able to model vocal fold approximation under the experimental conditions specified above.

(a)

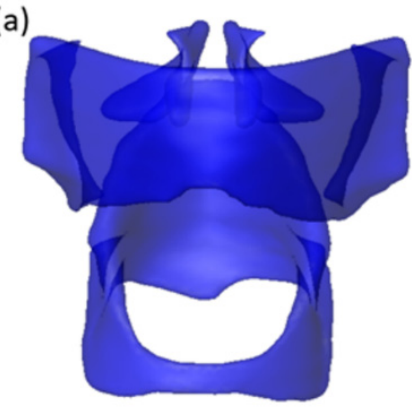

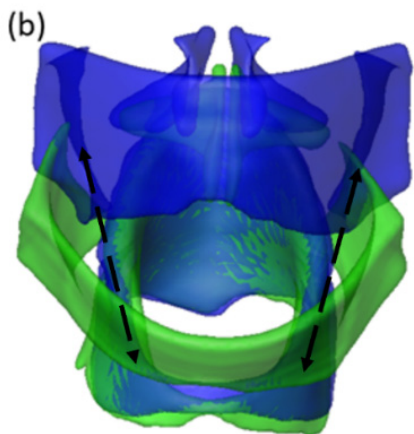

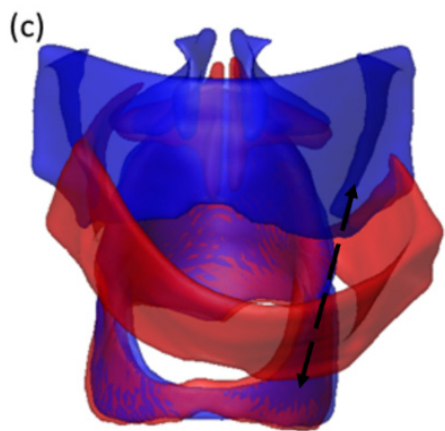

Figure 5. Reconstructed thyroid cartilage, arytenoid cartilages, and cricoid cartilage from MRI scans. (a) NA condition; (b) NA (blue) to HP (green) condition; (c) NA (blue) to sUVFP (red) condition. The arrows show the suture positions in (b) and (c). 


\section{Results}

\subsection{In Vivo Phonation Test Results}

HSV was captured for each subject in three conditions: NA, HP, and sUVFP. Figure 6 depicts the HSV analysis from subject 1 . Using a proprietary MATLAB ${ }^{\circledR}$ (Mathworks, Natick, MA) code generously provided by Dr. Dimitar Deliyski at Michigan State University and modified in-house, the HSV was converted for digital kymograph analysis (see Figure $6 b, c$ ), wherein a graphical representation of the vocal fold waveform with time as the $\mathrm{x}$-axis was generated. The HP condition (Figure $6 \mathrm{~b}$ ), demonstrated symmetrical frequency between the left and right vocal folds. The sUVFP condition demonstrated greater frequency on the sutured side (left) than the unsutured side (right).

(a)

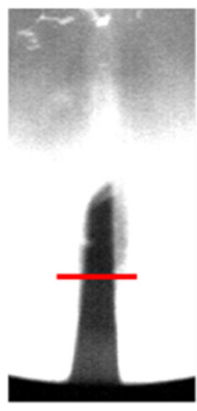

(b)

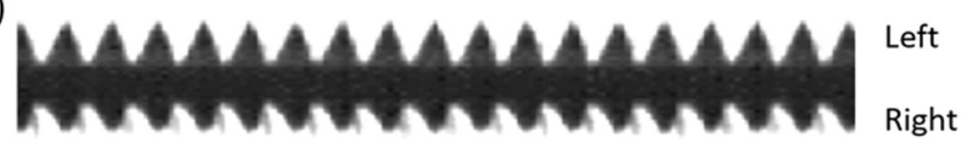

(c)

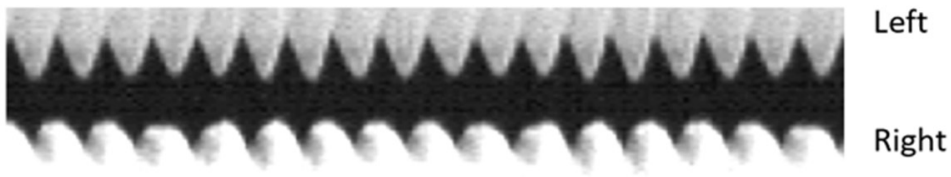

Figure 6. HSV analysis of Subject 1's vocal folds in a unilateral suture condition. (a) An isolated frame from HSV; the red line depicts the plane from which the kymograph was generated; (b) kymograph of the HP condition, same periodicity observed for left and right vocal folds; (c) kymograph of the sUVFP condition, the unsutured vocal fold (right) has fewer periods than the sutured vocal fold (left). Videos are included in the supplemental material.

Vibration frequencies calculated from HSV digital kymograph (Table 1) demonstrated predictable frequency differences between left and right vocal folds under the different experimental conditions. HP vocal folds achieved symmetrical vibration representative of normal phonation. Vocal folds with a unilateral suture (sUVFP) successfully modeled UVFP with asymmetrical vibration observed, resulting in a $10-11 \%$ difference in frequency between left and right vocal fold.

Table 1. Right and left vocal fold vibration frequencies obtained through HSV and digital kymography.

\begin{tabular}{ccccc}
\hline & & Right (Hz) & Left (Hz) & Difference \\
\hline \multirow{2}{*}{ Subject 1 } & HP & 875 & 875 & $<1 \%$ \\
& sUVFP & 815 & 908 & $11 \%$ \\
Subject 2 & HP & 660 & 660 & $<1 \%$ \\
\multirow{2}{*}{ Subject 3 } & sUVFP & 605 & 670 & $10 \%$ \\
& HP & 763 & 763 & $<1 \%$ \\
& sUVFP & 650 & 722 & $11 \%$ \\
\hline
\end{tabular}

\subsection{Eigenfrequency of Approximated Vocal Fold}

Using numerical methodology discussed in Section 2.2, subject-specific vocal fold approximation was modeled for different laryngeal conditions. During in vivo experiments, vibration frequency in the $\mathrm{HP}$ condition ranged from $500 \mathrm{~Hz}$ to $900 \mathrm{~Hz}$, consistent with the range observed in previous studies of rabbit phonation [30,37]. For computational modeling, we assumed constant material properties for the soft tissue including Young's moduli, Poisson's ratio, and density as listed in Table 2. The Young's modulus of the vocal fold cover used here falls in the range of previous experimental measurements $[57,58]$. The cartilages had negligible deformation and were thus assigned with much higher stiffness. These parameters would lead to a dominant eigenfrequency of around $600 \mathrm{~Hz}$ for the vocal fold in the HP condition. This frequency is consistent with the average frequency across the rabbit larynx samples in two previous studies from our group [29,30]. The vibration 
frequency in the present study had more variations among individuals. However, the differences between the experiment and computation did not impact analysis, as we sought to compare left-right vibratory frequency as opposed to making one-on-one comparisons between the two models.

Table 2. Tissue properties of cartilages, vocal fold body, and cover.

\begin{tabular}{cccc}
\hline & Young's Modulus (kPa) & Poisson's Ratio & Density $\left(\mathbf{k g} / \mathbf{m}^{\mathbf{3}}\right)$ \\
\hline Vocal fold body & 10 & 0.4 & 1000 \\
Vocal fold cover & 1 & 0.4 & 1000 \\
Cartilages & 500 & 0.4 & 1000 \\
\hline
\end{tabular}

A two-step process was used to compute the eigenfrequency of the approximated vocal fold. First, a static deformation was simulated for vocal fold adduction by the prescribed thyroid displacement according to the HP or sUVFP condition. As a result of this deformation, the vocal fold was pre-stressed. Then, the eigenfrequency and the eigenmode (or the vibration mode) were computed at the pre-stressed condition. Figure 7 shows vocal fold approximation under different conditions for Sample 1. Following reconstruction of the NA condition (Figure 7a), thyroid cartilage displacement for HP or sUVFP conditions was applied (Figure 7b or Figure 7c respectively). Using this subject-specific approach, we were able to model symmetrical approximation of the vocal folds for HP and asymmetrical approximation in sUVFP.

(a)

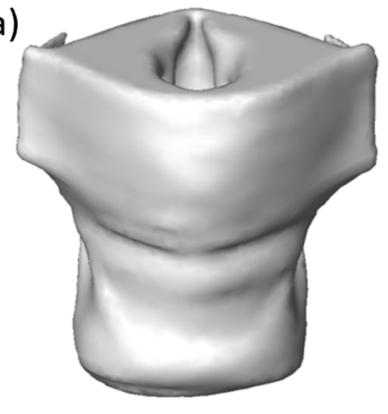

(b)

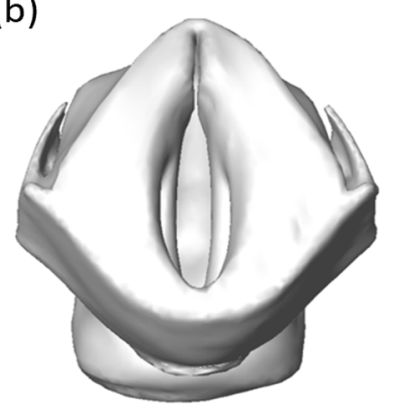

(c)

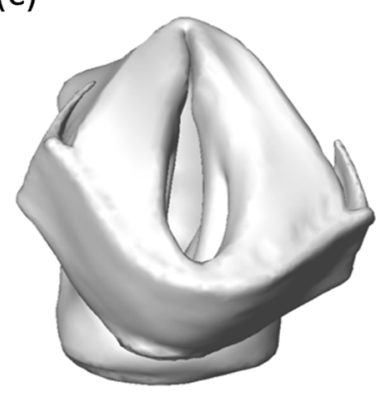

Figure 7. Reconstructed vocal fold under different approximation conditions for sample 1. (a) Released or NA, (b) two sides sutures or HP, (c) one side suture or sUVFP.

The eigenfrequency and eigenmode calculations were done using the default algorithm in COMSOL Multiphysics. Multiple modes were obtained from this simulation. The vocal vibration is mainly related to the first two modes for each side of the vocal fold [59]. The higher-frequency modes involve out-of-phase vibration patterns on the medial surface of the vocal fold and were not found from the HSV results (see supplementary material for those mode shapes and frequencies). The first mode has the maximal displacement in the axial direction (Y-axis in Figure 8), while the second mode has the maximal displacement in the direction transverse to the midline (X-axis in Figure 8$)$. The second mode was selected to represent the vibration pattern of the vocal fold under phonation by comparing it with the HSV results. This mode is shown in Figure 8 for sample 1. The corresponding eigenfrequency of the selected eigenmode is deemed the fundamental frequency of vibration. The eigenfrequencies for all three samples are listed in Table 3 for the NA, HP, and sUVFP conditions. The left and right sides are separately listed to compare the symmetry of vibration. From the results, the left and right sides had similar eigenfrequencies in the NA and HP conditions, while the sutured side had a 7-9\% higher eigenfrequency than the unsutured side in the sUVFP condition. Furthermore, both the NA and sUVFP conditions have much higher frequencies than the NA condition. The frequencies in the HP and sUVFP conditions show good agreement with the experimental findings in Section 3.1. 

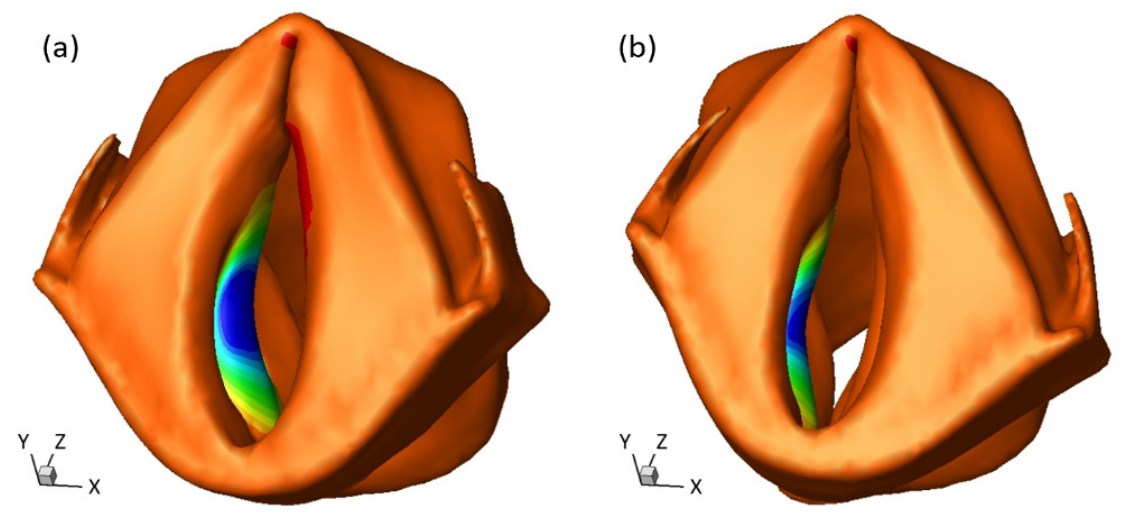

Figure 8. Eigenmodes of the vocal fold under different conditions. (a) HP condition with bilateral sutures (b) sUVFP condition with unilateral left-sided suture. Animations are included in the supplemental material.

Table 3. Left and right vocal fold eigenfrequencies under experimental conditions.

\begin{tabular}{ccccc}
\hline & & Right(Hz) & Left (Hz) & Difference \\
\hline \multirow{3}{*}{ Sample 1 } & NA & 334 & 333 & $<1 \%$ \\
& HP & 605 & 597 & $1 \%$ \\
& sUVFP & 560 & 610 & $9 \%$ \\
Sample 2 & NA & 302 & 300 & $<1 \%$ \\
& HP & 533 & 531 & $<1 \%$ \\
& sUVFP & 499 & 535 & $7 \%$ \\
Sample 3 & NA & 329 & 330 & $<1 \%$ \\
& HP & 609 & 608 & $<1 \%$ \\
& sUVFP & 506 & 550 & $9 \%$ \\
\hline
\end{tabular}

\section{Discussion}

In this study, in vivo phonation tests and subject-specific computation modeling were used to investigate the changes in vibratory frequency in three experimental conditions. We found a difference in vibratory patterns between $\mathrm{HP}$, as simulated by bilateral suture cricothyroid approximation, and SUVFP, as simulated by unilateral suture cricothyroid approximation. HP demonstrated symmetrical vibration while the sUVFP condition demonstrated asymmetry in left-right frequency. From this investigation, we concluded that the cricothyroid suture approximation approach implemented to generate the different laryngeal conditions in this experiment was valid to simulate both normal phonation and UVFP. The subjects demonstrated a wide range of vibration frequencies $(\sim 600-900 \mathrm{~Hz})$, as shown in Table 1; however, this variability is consistent with previous frequencies observed in non-stimulated and electrically stimulated phonation in rabbits $[29,30,39]$ and is likely attributable to inter-individual differences in tissue properties and laryngeal geometries. Given the high level of variability observed within species, one set of tissue properties will not adequately represent all subjects, illustrating a real need for subject-specific modeling to improve accuracy in surgical planning.

In the current sUVFP condition, the unsutured side's frequency was only around $10 \%$ lower than the sutured side, while both sides were much higher than the NA condition. This implies that the unsutured side in the sVUFP condition still experienced significant stretching and stiffening as a consequence of the contralateral suture. Further examination of the cartilage position in Figure 5 shows that both sides of the vocal fold were stretched even after one side's suture was released, and this was due to residual downward and lateral tilting of the released thyroid cartilage toward the contralateral suture. While the thyroid cartilage is flexible, it is a single piece of cartilage spanning the full breadth of the larynx, thus manipulation of one side cannot be achieved with total independence from the other. This type of asymmetric approximation is different than that observed in clinical 
UVFP; however, this technique successfully achieves significant differences between the left and right vocal fold and therefore creates a useful in vivo phonation paradigm that will allow us to perform future combined experimental/computational investigation into the surgical restoration of symmetric vibration.

The novel surgical and computational methods proposed in this study have several major benefits when compared to traditional techniques for modeling phonation and UVFP. Suture approximation is reversible and minimizes risks of airway patency, aspiration, and long-term survival associated with RLN/SLN resection in animal models [60]. Additionally, suture approximation allows for imaging of the vocal folds at rest, during bilateral phonation, and in sUVFP in a fast and minimally invasive process that can easily be replicated in vivo and ex vivo allowing for maximum translatability between the two. Using MRI scan and 3D reconstruction provides precise information about the cartilage movement during vocal fold adduction. With this information, the static deformation in the FEM simulation allows the model to account for the prestress produced by vocal fold adduction in the tissue. Thus, the subsequent eigenmode calculation captures the frequency and vibration mode of the approximated vocal fold in either bilateral adduction or unilateral adduction. Using this adduction-then-eigenmode simulation approach, we can estimate the vibration frequency without ad hoc assumptions for the tissue stiffening caused by the adduction, which may be generalized to calculate eigenfrequencies in the UVFP population. Future work will utilize this data to optimize surgical planning for type I thyroplasty, the gold standard surgical intervention in this disorder [2].

This study was limited in several ways. The inability to use the same subjects for HSV and anatomic imaging due to concerns for localized tissue changes following in vivo phonation impeded our ability to make direct comparisons of eigenfrequencies and vibratory frequencies within subjects. Thus, we adjusted for the lack of corresponding experimental frequency results by normalizing the eigenfrequency to an averaged frequency obtained from HSV in a sample outside of this study $(600 \mathrm{~Hz})$, which was lower than that observed in vivo during the surgical experiments in this study $(760 \mathrm{~Hz})$. As such, it is plausible that Young's moduli, Poisson's ratio, and tissue density have been overestimated or underestimated in individual subjects. Vocal fold vibration is essentially a fluid-structure interaction process between the glottal airflow and the soft tissue. A full 3D FSI simulation would provide the most accurate prediction, and we are working toward that direction with our current research. It has been demonstrated that the vocal fold vibration is highly related to its eigenfrequency $[59,61]$. Given the costly computational resources and time required for the high-fidelity simulations, utilizing the eigenfrequency model provides an efficient and meaningful way for such a combined experimental/computational initial investigation of the UVFP.

The use of ex vivo scanning limits translation of the present approach to human subjects, therefore future methods will rely on in vivo scans. Finally, while cricothyroid suture approximation shows promise for in vivo and ex vivo studies of vocal fold approximation and UVFP, variables such as suture placement and tension are hard to standardize, necessitating significant surgical skill, and this may result in lower precision than observed with other more established techniques (e.g., RLN resection to model UVFP, electrical stimulation for phonation.

\section{Conclusions}

In the present study, we describe a method to investigate vocal fold approximation in normal phonation and simulated UVFP conditions experimentally and numerically. Using a non-stimulated cricothyroid suture approximation model we previously developed to elicit sustained vocal fold phonation in rabbits, we implemented a novel experimental condition to simulate UVFP. Using this method, we demonstrated symmetrical phonation simulating normal phonation and asymmetrical phonation simulating UVFP.

A 3-dimensional MRI sequence was also performed in three experimental conditions: NA, HP, and sUVFP. These images were used to build a subject-specific vocal fold re- 
construction of laryngeal cartilages and two layers of soft tissue: vocal fold cover and vocal fold body. Using FEM, we simulated vocal fold deformation for each condition by incorporating the cartilage displacement. The subsequent eigenmode calculation accounts for the prestress in the tissue due to deformation. Similar to the surgical experimental results, the computational model also demonstrated similar eigenfrequencies between left and right in the HP condition and asymmetrical eigenfrequencies in the sUVFP condition.

The strong agreement between the changes in vibration frequency between conditions as observed in the HSV and the changes in eigenfrequency as calculated in the numerical analysis of the subject-specific model demonstrate that these novel methodologies are a promising innovation for the investigation of vocal fold phonation in healthy and UVFP conditions.

Supplementary Materials: The following are available online at https://www.mdpi.com/2076-341 7/11/4/1817/s1.

Author Contributions: The study was conceived by B.R. and H.L. Experiments were designed by Z.L., A.W., L.S., A.A., B.R. and H.L. Experimental data collection and analysis were performed by A.W. and L.S. Computational data collection was performed by Z.L., A.A. and H.L., Z.L., A.W., L.S., A.A., B.R. and H.L. contributed to the writing and critical revision of the manuscript. All authors have read and agreed to the published version of the manuscript.

Funding: This research was supported by NIH grant funding from the National Institute of Deafness and Other Communication Disorders (NIDCD) under Award Number 5 R01 DC016236 03 and the National Center For Advancing Translational Sciences of the National Institutes of Health (NCATS) under Award Number TL1TR001858. The content is solely the responsibility of the authors and does not necessarily represent the official views of the National Institutes of Health.

Institutional Review Board Statement: This study was approved by the University of Pittsburgh Institutional Animal Care and Use Committee (IACUC \#18114069). All procedures were conducted within the University of Pittsburgh Department of Laboratory Animal Research, an AAALACaccredited facility, in compliance with the Guide for the Care and Use of Laboratory Animals and the Animal Welfare Regulations.

Informed Consent Statement: Not applicable.

Data Availability Statement: The data presented in this study are available on request from the corresponding author. Final datasets will be publicly available when complete, per the resource sharing plan described in NIDCD award \#5R01DC016236.

Acknowledgments: We would like to thank Ye Chen and Yi Song for their contributions to computational modeling, Zach Zimmerman and Gary Gartling for their assistance with surgical procedures, and Kevin Hitchens and Lesley Foley for their professional guidance and technical assistance in MRI. The research reported in this study was supported by the National Institute on Deafness and Communication Disorders of the National Institute of Health under award number: 5R01DC016236, PI: Rousseau. The content is solely the responsibility of the authors and does not necessarily represent the official views of the National Institutes of Health.

Conflicts of Interest: The authors declare no conflict of interest in the present study.

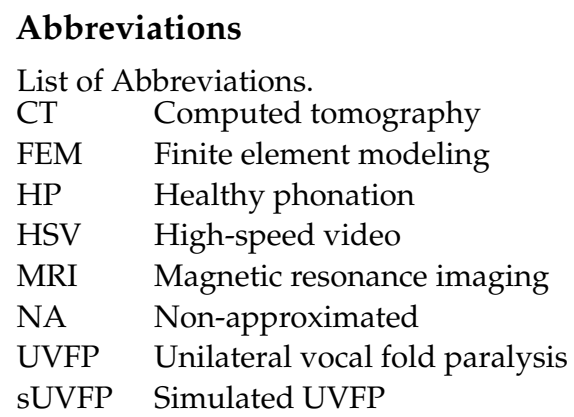




\section{References}

1. Titze, I.R.; Jiang, J.; Drucker, D.G. Preliminaries to the body-cover theory of pitch control. J. Voice 1988, 1, 314-319. [CrossRef]

2. Wilson, A.; Kimball, E.E.; Sayce, L.; Luo, H.; Khosla, S.M.; Rousseau, B. Medialization Laryngoplasty: A Review for SpeechLanguage Pathologists. J. Speech Lang. Hear. Res. 2021, 1-10. [CrossRef]

3. Havas, T.; Lowinger, D.; Priestley, J. Unilateral Vocal Fold Paralysis: Causes, Options and Outcomes. ANZ J. Surg. 1999, 69, 509-513. [CrossRef]

4. Rosenthal, L.H.S.; Benninger, M.S.; Deeb, R.H. Vocal Fold Immobility: A Longitudinal Analysis of Etiology Over 20 Years. Laryngoscope 2007, 117, 1864-1870. [CrossRef]

5. Sulica, L. The Natural History of Idiopathic Unilateral Vocal Fold Paralysis: Evidence and Problems. Laryngoscope 2008, 118, 1303-1307. [CrossRef]

6. Francis, D.O.; McKiever, M.E.; Garrett, C.G.; Jacobson, B.; Penson, D.F. Assessment of patient experience with unilateral vocal fold immobility: A preliminary study. J. Voice 2014, 28, 636-643. [CrossRef]

7. Kelchner, L.N.; Stemple, J.C.; Gerdeman, B.; Le Borgne, W.; Adam, S. Etiology, pathophysiology, treatment choices, and voice results for unilateral adductor vocal fold paralysis: A 3-year retrospective. J. Voice 1999, 13, 592-601. [CrossRef]

8. Paniello, R.C.; Edgar, J.D.; Kallogjeri, D.; Piccirillo, J.F. Medialization versus reinnervation for unilateral vocal fold paralysis: A multicenter randomized clinical trial. Laryngoscope 2011, 121, 2172-2179. [CrossRef] [PubMed]

9. Brunner, E.; Friedrich, G.; Kiesler, K.; Chibidziura-Priesching, J.; Gugatschka, M. Subjective Breathing Impairment in Unilateral Vocal Fold Paralysis. Folia Phoniatr. Logop. 2011, 63, 142-146. [CrossRef]

10. Francis, D.O.; Ms, K.W.; Hovis, K.; Gelbard, A.; Merati, A.L.; Penson, D.F.; Netterville, J.L.; Garrett, C.G. Effect of injection augmentation on need for framework surgery in unilateral vocal fold paralysis. Laryngoscope 2016, 126, 128-134. [CrossRef] [PubMed]

11. Leder, S.B.; Suiter, D.M.; Duffey, D.; Judson, B.L. Vocal Fold Immobility and Aspiration Status: A Direct Replication Study. Dysphagia 2011, 27, 265-270. [CrossRef]

12. Bertroche, J.T.; Radder, M.; Kallogjeri, D.; Paniello, R.C.; Bradley, J.P. Patient-defined duration of benefit from juvederm (hyaluronic acid) used in injection laryngoplasty. Laryngoscope 2019, 129, 2744-2747. [CrossRef] [PubMed]

13. Cohen, J.T.; Benyamini, L. Voice outcome after vocal fold injection augmentation with carboxymethyl cellulose versus calcium hydroxyapatite. J. Laryngol. Otol. 2020, 134, 263-269. [CrossRef]

14. Fang, T.-J.; Li, H.-Y.; Gliklich, R.E.; Chen, Y.-H.; Wang, P.-C.; Chuang, H.-F. Outcomes of Fat Injection Laryngoplasty in Unilateral Vocal Cord Paralysis. Arch. Otolaryngol. Head Neck Surg. 2010, 136, 457. [CrossRef] [PubMed]

15. Kwon, T.-K.; Rosen, C.A.; Gartner-Schmidt, J. Preliminary Results of a New Temporary Vocal Fold Injection Material. J. Voice 2005, 19, 668-673. [CrossRef]

16. Mallur, P.S.; Morrison, M.P.; Postma, G.N.; Amin, M.R.; Rosen, C.A. Safety and efficacy of carboxymethylcellulose in the treatment of glottic insufficiency. Laryngoscope 2012, 122, 322-326. [CrossRef] [PubMed]

17. Zeitels, S.M.; Lombardo, P.J.; Chaves, J.L.; Faquin, W.C.; Hillman, R.E.; Heaton, J.T.; Kobler, J.B. Vocal Fold Injection of Absorbable Materials: A Histologic Analysis with Clinical Ramifications. Ann. Otol. Rhinol. Laryngol. 2019, 128, 71S-81S. [CrossRef] [PubMed]

18. Zeleník, K.; Walderová, R.; Kučová, H.; Jančatová, D.; Komínek, P. Comparison of long-term voice outcomes after vocal fold augmentation using autologous fat injection by direct microlaryngoscopy versus office-based calcium hydroxylapatite injection. Eur. Arch. Oto Rhinol. Laryngol. 2017, 274, 3147-3151. [CrossRef]

19. Cohen, J.T.; Bates, D.D.; Postma, G.N. Revision Gore-Tex Medialization Laryngoplasty. Otolaryngol. Neck Surg. 2004, 131, 236-240. [CrossRef] [PubMed]

20. Maragos, N.E. Revision Thyroplasty. Ann. Otol. Rhinol. Laryngol. 2001, 110, 1087-1092. [CrossRef]

21. Netterville, J.L.; Stone, R.E.; Civantos, F.J.; Luken, E.S.; Ossoff, R.H. Silastic Medialization and Arytenoid Adduction: The Vanderbilt Experience. Ann. Otol. Rhinol. Laryngol. 1993, 102, 413-424. [CrossRef]

22. Parker, N.P.; Barbu, A.M.; Hillman, R.E.; Zeitels, S.M.; Burns, J.A. Revision Transcervical Medialization Laryngoplasty for Unilateral Vocal Fold Paralysis. Otolaryngol. Neck Surg. 2015, 153, 593-598. [CrossRef] [PubMed]

23. Woo, P.; Pearl, A.W.; Hsiung, M.-W.; Som, P. Failed medialization laryngoplasty: Management by revision surgery. Otolaryngol. Neck Surg. 2001, 124, 615-621. [CrossRef] [PubMed]

24. Green, D.C.; Berke, G.S.; Ward, P.H. Vocal Fold Medialization by Surgical Augmentation versus Arytenoid Adduction in the in vivo Canine Model. Ann. Otol. Rhinol. Laryngol. 1991, 100, 280-287. [CrossRef]

25. Hiroto, I. Surgical voice improvement for unilateral recurrent laryngeal nerve paralysis. Otologia 1976, 22, 473-474.

26. Orestes, M.I.; Neubauer, J.; Sofer, E.; Salinas, J.; Chhetri, D.K. Phonatory effects of type I thyroplasty implant shape and depth of medialization in unilateral vocal fold paralysis. Laryngoscope 2014, 124, 2791-2796. [CrossRef] [PubMed]

27. Nasseri, S.S.; Maragos, N.E. Combination thyroplasty and the "twisted larynx:" combined type IV and type I thyroplasty for superior laryngeal nerve weakness. J. Voice 2000, 14, 104-111. [CrossRef]

28. Shaw, G.Y.; Searl, J.P.; Hoover, L.A. Diagnosis and treatment of unilateral cricothyroid muscle paralysis with a modified Isshiki type 4 thyroplasty. Otolaryngol. Neck Surg. 1995, 113, 679-688. [CrossRef]

29. Novaleski, C.K.; Kojima, T.; Chang, S.; Luo, H.; Valenzuela, C.V.; Rousseau, B. Nonstimulated rabbit phonation model: Cricothyroid approximation. Laryngoscope 2016, 126, 1589-1594. [CrossRef] [PubMed] 
30. Chang, S.; Novaleski, C.K.; Kojima, T.; Mizuta, M.; Luo, H.; Rousseau, B. Subject-Specific Computational Modeling of Evoked Rabbit Phonation. J. Biomech. Eng. 2016, 138, 011005-0110056. [CrossRef] [PubMed]

31. Chang, S.; Tian, F.-B.; Luo, H.; Doyle, J.F.; Rousseau, B. The Role of Finite Displacements in Vocal Fold Modeling. J. Biomech. Eng. 2013, 135, 111008. [CrossRef]

32. Li, Z.; Chen, Y.; Chang, S.; Luo, H. A Reduced-Order Flow Model for Fluid-Structure Interaction Simulation of Vocal Fold Vibration. J. Biomech. Eng. 2020, 142, 0210051-02100510. [CrossRef]

33. Luo, H.; Mittal, R.; Bielamowicz, S.A. Analysis of flow-structure interaction in the larynx during phonation using an immersedboundary method. J. Acoust. Soc. Am. 2009, 126, 816-824. [CrossRef]

34. Sadeghi, H.; Kniesburges, S.; Falk, S.; Kaltenbacher, M.; Schützenberger, A.; Döllinger, M. Towards a Clinically Applicable Computational Larynx Model. Appl. Sci. 2019, 9, 2288. [CrossRef]

35. Xue, Q.; Zheng, X.; Mittal, R.; Bielamowicz, S. Subject-specific computational modeling of human phonation. J. Acoust. Soc. Am. 2014, 135, 1445-1456. [CrossRef] [PubMed]

36. Yang, J.; Wang, X.; Krane, M.; Zhang, L.T. Fully-coupled aeroelastic simulation with fluid compressibility-For application to vocal fold vibration. Comput. Methods Appl. Mech. Eng. 2017, 315, 584-606. [CrossRef]

37. Chen, Y.; Li, Z.; Chang, S.; Rousseau, B.; Luo, H. A reduced-order flow model for vocal fold vibration: From idealized to subject-specific models. J. Fluids Struct. 2020, 94, 102940. [CrossRef] [PubMed]

38. Mittal, R.; Erath, B.D.; Plesniak, M.W. Fluid Dynamics of Human Phonation and Speech. Annu. Rev. Fluid Mech. 2013, 45, 437-467. [CrossRef]

39. Swanson, E.R.; Ohno, T.; Abdollahian, D.; Rousseau, B. Effects of raised-intensity phonation on inflammatory mediator gene expression in normal rabbit vocal fold. Otolaryngol. Neck Surg. 2010, 143, 567-572. [CrossRef]

40. Alipour, F.; Brücker, C.; Cook, D.D.; Gömmel, A.; Kaltenbacher, M.; Mattheus, W.; Mongeau, L.; Nauman, E.; Schwarze, R.; Tokuda, I.; et al. Mathematical Models and Numerical Schemes for the Simulation of Human Phonation. Curr. Bioinform. 2011, 6, 323-343. [CrossRef]

41. Zhang, Z.; Kreiman, J.; Gerratt, B.R.; Garellek, M. Acoustic and perceptual effects of changes in body layer stiffness in symmetric and asymmetric vocal fold modelsa. J. Acoust. Soc. Am. 2013, 133, 453-462. [CrossRef] [PubMed]

42. Birk, V.; Kniesburges, S.; Semmler, M.; Berry, D.A.; Bohr, C.; Döllinger, M.; Schützenberger, A. Influence of glottal closure on the phonatory process in ex vivo porcine larynges. J. Acoust. Soc. Am. 2017, 142, 2197-2207. [CrossRef]

43. Döllinger, M.; Berry, D.A.; Kniesburges, S. Dynamic vocal fold parameters with changing adduction inex-vivohemilarynx experiments. J. Acoust. Soc. Am. 2016, 139, 2372-2385. [CrossRef] [PubMed]

44. Goodyer, E.; Müller, F.; Licht, K.; Hess, M. In vivo measurement of the shear modulus of the human vocal fold: Interim results from eight patients. Eur. Arch. Oto Rhino. Laryngol. 2007, 264, 631-635. [CrossRef]

45. Hadwin, P.J.; Motie-Shirazi, M.; Erath, B.D.; Peterson, S.D. Bayesian Inference of Vocal Fold Material Properties from Glottal Area Waveforms Using a 2D Finite Element Model. Appl. Sci. 2019, 9, 2735. [CrossRef]

46. Hunter, E.J.; Titze, I.R.; Alipour, F. A three-dimensional model of vocal fold abduction/adduction. J. Acoust. Soc. Am. 2004, 115, 1747-1759. [CrossRef]

47. Lamprecht, R.; Maghzinajafabadi, M.; Semmler, M.; Sutor, A. Imaging the Vocal Folds: A Feasibility Study on Strain Imaging and Elastography of Porcine Vocal Folds. Appl. Sci. 2019, 9, 2729. [CrossRef]

48. Maghzinajafabadi, M.; Lamprecht, R.; Sutor, M.S.A.A.; Semmler, M.; Sutor, A. Acoustic Pressure Pipette Aspiration Method Combined with Finite Element Analysis for Isotropic Materials. Appl. Sci. 2019, 9, 3875. [CrossRef]

49. Chen, T.; Chodara, A.M.; Sprecher, A.J.; Fang, F.; Song, W.; Tao, C.; Jiang, J.J. A New Method of Reconstructing the Human Laryngeal Architecture Using Micro-MRI. J. Voice 2012, 26, 555-562. [CrossRef]

50. Hoffman, M.R.; Glab, R.; Gunderson, M.; Maytag, A.L.; Yang, D.T.; Jiang, J.J.; Dailey, S.H. Functional and Histological Evaluation following Canine Vocal Fold Reconstruction Using Composite Thyroid Ala Perichondrium Flaps. Otolaryngol. Neck Surg. 2015, 153, 79-87. [CrossRef]

51. U.S. Department of Agriculture. Animal Care: Animal Welfare Act and Animal Welfare Regulations. (n.d.). Available online: www.ascr.usda.gov / fling-program-discrimination (accessed on 31 December 2020).

52. National Research Council. Guide for the Care and Use of Laboratory Animals; National Academies Press: Cambridge, MA, USA, 2011.

53. Sayce, L.J.; E Powell, M.; E Kimball, E.; Chen, P.; Gartling, G.J.; Rousseau, B. Continuous Rate Infusion of Ketamine Hydrochloride and Dexmedetomidine for Maintenance of Anesthesia during Laryngotracheal Surgery in New Zealand White Rabbits (Oryctolagus cuniculus). J. Am. Assoc. Lab. Anim. Sci. 2020, 59, 176-185. [CrossRef]

54. Hirano, M. Phonosurgery. Basic and clinical investigations. Otology (Fukuoka) 1975, 21, 239-242.

55. Tian, F.-B.; Dai, H.; Luo, H.; Doyle, J.F.; Rousseau, B. Fluid-structure interaction involving large deformations: 3D simulations and applications to biological systems. J. Comput. Phys. 2014, 258, 451-469. [CrossRef]

56. Alipour, F.; Berry, D.A.; Titze, I.R. A finite-element model of vocal-fold vibration. J. Acoust. Soc. Am. 2000, 108, 3003-3012. [CrossRef]

57. Rousseau, B.; Hirano, S.; Chan, R.W.; Welham, N.V.; Thibeault, S.L.; Ford, C.N.; Bless, D.M. Characterization of chronic vocal fold scarring in a rabbit model. J. Voice 2004, 18, 116-124. [CrossRef] [PubMed] 
58. Thibeault, S.L.; Gray, S.D.; Bless, D.M.; Chan, R.W.; Ford, C.N. Histologic and rheologic characterization of vocal fold scarring. J. Voice 2002, 16, 96-104. [CrossRef]

59. Yin, J.; Zhang, Z. The influence of thyroarytenoid and cricothyroid muscle activation on vocal fold stiffness and eigenfrequencies. J. Acoust. Soc. Am. 2013, 133, 2972-2983. [CrossRef]

60. Caballero, M.; Bernal-Sprekelsen, M.; Calvo, C.; Farré, X.; Alós, L. Autologous elastic cartilage for laryngoplasty: Histologic evaluation in a rabbit model. Ann. Otol. Rhinol. Laryngol. 2003, 112, 734-739. [CrossRef] [PubMed]

61. Berry, D.A. Mechanisms of modal and nonmodal phonation. J. Phon. 2001, 29, 431-450. [CrossRef] 